Andrea Mennicken Hendrik Vollmer (Hrsg.)

Zahlenwerk

Kalkulation, Organisation und Gesellschaft 


\title{
Zahlenspiele in der Entwicklungshilfe: Zu einer Soziologie des Deckungsbeitrages ${ }^{1}$
}

\author{
Stefan Kühl
}

Wäre Entwicklungshilfe ein Geschenk würden Zahlen eine untergeordnete Rolle spieien. Zugegeben: Man könnte messen, ob das Geschenk des einen Industrielandes an ein Entwicklungsland größer ist als das Geschenk eines anderen Industrielandes. Man könnte feststellen, ob das Geschenk, das ein Ölstaat vergibt, größer ist als im vorigen Jahr. Oder man könnte versuchen herauszubekommen, wie hoch die Gesamtsumme von erhaltenen Geschenken aus den Industriestaaten ist. Aber die Quantifizierung von Geschenken ist - jedenfalls in modernen Gesellschaften - weitgehend tabuisiert. Gegenstände und Dienstleistungen scheinen sich einer Quantifizierung ihres Wertes, ihres Nutzens zu entziehen, wenn sie die Form eines Geschenkes annehmen (vgl. zur Funktion des Schenkens Mauss 1990). Man sieht es an der Tabuisierung des Vergleichs zwischen Schenkenden. Man merkt es, wenn der Beschenkte nur verschämt abzuschätzen wagt, ob der eigene Lebenspartner bei den Weihnachtsgeschenken für einen mal wieder gespart hat. Aber Entwicklungshilfe ist kein Geschenk. Häufig wird Entwicklungshilfe nur als - wenn auch subventionierter Kredit vergeben. Sowohl bei der Vergabe der Entwicklungshilfe als Kredit als auch als Zuschuss wird diese an Bedingungen geknüpft, beispielsweise die Verabschiedung von Gesetzen oder die Verlagerung von Schwerpunkten in Budgets. Weiterhin wird - auch wegen den Rechenschaftspflichten der großen Geberorganisationen - genau spezifiziert, was mit der Entwicklungshilfe gemacht und erreicht werden soll.

Weil die Entwicklungshilfe in der Regel an eine Vielzahl von Bedingungen geknüpft wird, spielen Zahlen eine zentrale Rolle. Die Berechtigung für Entwicklungshilfe wird zum Beispiel darin gesehen, dass das Durchschnittseinkommen der Bevölkerung unter einem festgesetzten Entwicklungshilfebetrag liegt. Die Freigabe von Mitteln wird häufig daran gebunden, dass die Empfängerorganisationen ein genau definiertes Jahrergebnis erzielen. Der Erfolg der Entwicklungshilfe wird darin gemessen, inwiefern sich quantitative, häufig monetäre Indikatoren verbessert haben. In den Hochglanzbroschüren der Entwicklungshilfeorganisationen mögen (je nach Intention) weinende oder glücklich lächelnde Kinder gezeigt werden, eine Versammlung eifrig diskutierender Dorfbewohner abgebildet oder Zwei-Kammer-Toiletten als die neuste technischen Errungenschaft präsentiert werden, aber die V.erständigung zwischen verschiedenen in der Entwicklungshilfe aktiven Organisationen läuft ganz maßgeblich über Zahlen. Zahlen sind die Verkehrsprache, mit denen Organisationen aus ganz unterschiedlichen Kulturen versuchen sich zu verständigen. Es spricht vieles dafür, dass Zahlen die „Lingua Franca“ sind, mit denen sich in der Entwicklungshilfe

i Ich danke Andrea Mennicken und Hendrik Vollmer für die ausführliche Kommentierung einer früheren Fassung des Artikels. 
verständigt wird. ${ }^{2}$ Dabei können wir uns ganz verschiedene Formen von Zahlen vorstellen: die Prozentzahl von Personen, die Zugang zu einer Basisgesundheitsversorgung haben, die Kilometer, die ein Dorfbewohner durchschnittlich zur nächsten Straße gehen muss, die Absolventenzahlen nach Einführung eines Bildungsprogramms oder die Prozentzahl des Wassers, das durch lecke Rohre versickert (vgl. z.B. Wisner 1989: 47),

Zahlen erscheinen auf den ersten Blick als neutral, unabhängig und objektiv und signalisieren so, dass es kaum Interpretationsspielraum gibt (vgl. Porter 1995: x). Eine Zahlenangabe, so wenigstens die erste Suggestion, repräsentiert in einem westeuropäischen Land das gleiche wie in einem Land in Subsahara-Afrika. Eine Gewinn-und-Verlust-Rechnung funktioniere, so die Annahme, in einem US-amerikanischen Unternehmen genauso wie bei seinem philippinischen Kooperationspartner und ist von regionalen oder kulturellen Besonderheiten unabhängig. Aber auch bei dieser „Lingua Franca“" kann es Dialekte geben. Bei Dialekten handelt es sich im Verständnis der Linguistik um Varietäten einer Sprache. Sprachen und ihre Dialekte sind dabei von ihrer Grundstruktur so eng gekoppelt, dass sich der Sprecher einer Hochsprache und die Sprecher eines Dialektes miteinander verständigen können. Für Zahlen als „Lingua Franca“ bedeutet dies, dass zwar im Rahmen eines weltweiten Standards unterschiedliche Berechnungsformen existieren, aber die konkurrierenden Berechnungsformen verstanden werden. ${ }^{3}$

Inzwischen gibt es in der soziologisch orientierten Accounting-Forschung eine Reihe von Studien, die die Ausbildung unterschiedlicher „Zahlendialekte“ sehr detailliert herausgearbeitet haben. ${ }^{4}$ So wurde beispielsweise in Studien über Instandhaltungseinheiten der US-amerikanischen Armee (vgl. Ansari/Euske 1987), australische Krankenhäuser (vgl. Chua 1995), deutsche und britische Brauereien (Ahrens 1996; Ahrens 1997), multinationale Accounting-Firmen Osteuropas (vgl. Cooper et al. 1998), Forschungs- und Entwicklungsabteilungen (vgl. Robson 1993), internationale Hotelketten (Gustavsson 2000) und den britischen National Health Service (vgl. Pinch et al. 2000) dargestellt, wie Zahlen durch unterschiedliche Sinnzusammenhänge geprägt werden (siehe auch die Sammelbände von Hopwood/Miller 1994 und Kalthoff et al. 2000).

Das Ziel dieses Artikels ist es - anknüpfend an diese Forschungen - Zahlendialekte in den Kooperationsbeziehungen zwischen nationalen und internationalen Entwicklungshilfeorganisationen, staatlichen und privaten Einrichtungen in Entwicklungsländern und den in der Entwicklungshilfe tätigen Beratungsorganisationen näher zu analysieren. Der Artikel fokussiert besonders auf die Verwendung monetär gefasster Kennzahlen in der Entwick-

2 In der Analyse von Accounting haben Metaphern der Sprache eine gewisse Tradition. Siehe z.B. Tinker et al. 1988; Robson 1991; Munro 1993.

3 In der Linguistik ist die Unterscheidung zwischen Sprache und Dialekt umstritten, weil Sprache eine Höherwertigkeit gegenüber Dialekten signalisiert und die Bestimmung als Sprache oder Dialekt häufig von politischen Rahmenbedingungen abhängt.

4 Soziologisch orientierte Accounting-Forschung ist zugegebenermaßen ein unpräziser Begriff. Er umfasst vorrangig deskriptiv, nicht normativ ausgerichtete Ansätze. Diese Ansätze werden mit so unterschiedlichen Begriffen wie „Behavioral Accounting“ (vgl. Hopwood 1987) oder „Critical Accounting“ (vgl. Dillard 1991) oder „Radical Accounting“" (vgl. Hunt/Hogler 1990) bezeichnet. Dazu können besonders ethnomethodologische (vgl. Chua 1995), neoinstitutionalistische (vgl. Meyer 1986), marxistische (vgl. Tinker et al. 1988), systemtheoretische (vgl. Scheytt 2005) und an Foucault orientierte Ansätze (vgl. Rose 1990; Hacking 1990) gezählt werden. Hendrik Vollmer (2004: 454) macht darauf aufmerksam, dass das Zugeständnis wirklichkeitskonstitutierender Effekte organisierten Rechnens als Minimalkonsens dieser Schulen zu betrachten ist und darüber die Abgrenzung zum wirtschafts- und managementwissenschaftlichen Mainstream vorgenommen wird. 
lungszusammenarbeit. Ökonomische Kennziffern können dabei als eine Sonderform von Zahlen begriffen werden, die dann entstehen, wenn soziale Phänomene in Geldbeträgen quantifiziert werden. Dabei ist der Übergang zwischen den nicht in Geldbeträgen gefasste Zahlen und den in Geldbeträgen gefasste Zahlen fließend (Munro 1993). Jedes soziales Phänomen lässt sich - mehr oder minder gut - in Zahlen fassen und jede nichtmonetäre Zahl lässt sich in Dollar-, Euro- oder Pfundbeträge überführen. Die Bezifferung der ökonomischen Verluste durch eine hohe HIV-Erkrankungsrate in Dollar, Euro oder Rand ist häufig noch eindrucksvoller als die reine Prozentzahl von AIDS-Kranken. Die Übersetzung der in Litern ausgedrückten technischen Wasserverluste einer nationalen Wasserbehörde in Peso, Rupie oder Riyal verleiht der in Geld ausgedrückten Zahl eine eigene Nachdrücklichkeit. ${ }^{5}$

Monetäre Zahlen greifen - im Gegensatz zu anderen Zahlen wie Lehrauslastung, Krankheitszeiten oder Überstunden - auf den Charakter von Geld als symbolisch generalisiertes Kommunikationsmedien zurück (vgl. speziell zu Geld Luhmann 1972: 187ff; Luhmann 1981: 397; Luhmann 1988: 306; allgemein zu symbolisch generalisierten Kommunikationsmedien Luhmann 1997: 316ff). Geld erhöht - ähnlich wie in anderen Kontexten Macht, Liebe oder Wahrheit - die Erfolgswahrscheinlichkeit von Kommunikationen. Man ist als Berater bereit mit Projektmanagern einer staatlichen Beratungsorganisation Zeit zu verbringen, weil man mit Geld (oder in sehr seltenen Fällen alternativ durch Liebe oder Macht) dazu gebracht wird. Große multinationale Entwicklungshilfeorganisationen nutzen das in Aussichtstellen größerer Geldbeträge dazu, um in Entwicklungsländern Veränderungen im Staatshaushalt durchzusetzen. Durch die Fassung in symbolisch generalisierte Kommunikationsmedien werden soziale Phänomene vergleichbar. Ein soziales Phänomen, das als Geldsumme gefasst ist, kann mit fast jedem beliebigen anderen sozialen Phänomen verglichen werden. Durch diese Vergleichbarkeit erhält eine Zahl eine ganz eigene Form von Aufmerksamkeit. Ein Vertreter der Weltbank kann das Bruttoinlandprodukt von Senegal in der Höhe von 5 Milliarden US-Dollar mit dem Umsatz von General Motors von 200 Milliarden US-Dollar vergleichen. Eine 12-jährige Schülerin in Washington kann das durchschnittliche Tageseinkommen in Nigeria von $1 \$$ mit dem eigenen Taschengeld in Beziehung setzen. Der Erfolg eines Beratungsprojektes kann durch die Berater in Geldbeträge gefasst werden und erscheint dann plötzlich gegenüber dem aufgewandten Beraterhonorar überraschend günstig.

Im Mittelpunkt des Artikels wird eine einzige ökonomische Kennzahl gestellt: der Deckungsbeitrag. Diese Kennzahl bestimmt sich aus der Differenz zwischen den Geschäftseinnahmen (zum Beispiel durch den Verkauf von Gütern) und den Ausgaben für das laufende Geschäft (z.B. für Rohstoffe, Ersatzteile oder Personal). Über die - nur auf den

5 Besten Dank an Hendrik Vollmer für einige Hinweise zur besonderen Charakterisierung ökonomischer Kennziffern. Von einer eindrucksvollen Übersetzung berichten Neu und Ocampo (2006) in ihrem Artikel über die Bildungspolitik der Weltbank in Latein Amerika. Bei der Definition eines Projektes in Panama wird beispielsweise die "rate of return" von $22 \%$ genau bestimmt. Die Rate basiert auf einer geschätzten Einkommenssteigerung, die die Kinder aufgrund einer verbesserten Bildung erhalten würden. Dies wird im Weltbank-Dokument genauer ausgefuhrt. „Throughout the calculation, the maintained hypothesis was a working life of 45 years. Yearly wages for different education levels were estimated from the 1997 Living Standard Measurement Survey ... To estimate the expected yearly income, the unemployment rates for the category "All Poor" were estimated from the 1997 LSMS data. The unemployment rate for people who finished lower secondary education is substantially higher than for people with a less educated background. ... To incorporate these in the calculations, the unemployment rate for someone with a lower secondary education was taken to be 2.5 times that of a primary educated person, but to decline linearly by .1 percent point per year of working life until both unemployment rates reach the same level." 
ersten Blick - dröge wirkende Kennziffer des Deckungsbeitrages versuchen Entwicklungshilfeorganisationen Organisationen in verschiedenen Entwicklungsländern zu steuern. So wird häufig die Auszahlung der Entwicklungshilfe an eine Verbesserung des Deckungsbeitrages knüpfen. Auch die Honorierung von Beratungsunternehmen oder von durch die Entwicklungshilfe finanzierten privaten Betreiberunternehmen wird zunehmend an einer Verbesserung des Deckungsbeitrages abhängig gemacht. ${ }^{6}$

\begin{tabular}{|c|c|c|}
\hline Wasserversorgungsbetreiber & Ökonomische Situation & Betreibermodell \\
\hline Im Norden des Landes & $\begin{array}{l}\text { Versorgt vorrangig die } \\
\text { Hauptstadt mit Wasser. Es } \\
\text { gibt eine Vielzahl von pri- } \\
\text { vaten und industriellen } \\
\text { Anschlüssen. Wegen der } \\
\text { Ballung im städtischen } \\
\text { Raum sind die Erschlie- } \\
\text { Bungs- und Wartungskosten } \\
\text { relativ gering. }\end{array}$ & $\begin{array}{l}\text { Seit einigen Jahren wird } \\
\text { die Wasserversorgung } \\
\text { durch ein Konsortium um } \\
\text { ein internationales Was- } \\
\text { serunternehmen betrie- } \\
\text { ben. }\end{array}$ \\
\hline Im Westen des Landes & $\begin{array}{l}\text { Versorgt neben privaten } \\
\text { Haushalten vorrangig eine } \\
\text { zweistellige Zahl von Un- } \\
\text { ternehmen im zentralen } \\
\text { Hafen des Landes. Weil es } \\
\text { sich bei diesen Unterneh- } \\
\text { men um zuverlässig zah- } \\
\text { lende Großabnehmern han- } \\
\text { delt, kann das Unternehmen } \\
\text { kostendeckend arbeiten. }\end{array}$ & $\begin{array}{l}\text { Seit einigen Jahren wird } \\
\text { die Wasserversorgung } \\
\text { durch ein staatliches } \\
\text { Unternehmen betrieben. } \\
\text { Dieses Unternehmen ist } \\
\text { im Besitz mehrerer unter- } \\
\text { schiedlicher staatlicher } \\
\text { Institutionen. }\end{array}$ \\
\hline Im Osten des Landes & $\begin{array}{l}\text { Versorgt werden vorrangig } \\
\text { mittelgroße Städte im länd- } \\
\text { lichen Raum sowie einige } \\
\text { kleinere Ansiedlungen. }\end{array}$ & $\begin{array}{l}\text { Die Wasserversorgung } \\
\text { wird durch eine Unterbe- } \\
\text { hörde der zentralen nati- } \\
\text { onalen Wasserbehörde } \\
\text { betrieben. Ziel ist es, } \\
\text { diese Wasserbehörde } \\
\text { mittelfristig in den Status } \\
\text { eines staatlichen Unter- } \\
\text { nehmens zu überführen. }\end{array}$ \\
\hline
\end{tabular}

Als Empirie für diesen Artikel dienen mir die „Zahlenspiele“, die zwischen großen nationalen und multinationalen Entwicklungshilfeorganisationen und drei größeren Wasserversorgungsbetreibern in einem afrikanischen Land stattfinden. ${ }^{7}$ Die drei untersuchten größeren

6 Aus meiner Sicht lassen sich politische Prozesse um Accounting besonders gut anhand von bestimmten ökonomischen Berechnungsverfahren bestimmen. Siehe zum Beispiel Powers (1992) Studie über die Frage, wie der Wert yon Markennamen zu bestimmen ist.

7 Über insgesamt einen Monat wurden Experteninterviews mit insgesamt 41 Mitarbeitern von internationalen und nationalen Entwicklungshilfeorganisationen (sieben Gesprächspartner), des zuständigen Ministerium (vier Gesprächspartner inklusive Minister und Staatssekretär), der nationalen Wasserbehörde (neun Gesprächspartner), der drei regionalen Wasserversorgungseinheiten (15 Gesprächspartner) und der engagierten 
Wasserunternehmen, die alle aus einer zentralen nationalen Wasserbehörde hervorgegangen sind, unterscheiden sich grundlegend voneinander. Das Wasserunternehmen im Norden des Landes, das unter anderem die Metropole des Landes versorgt, wird seit einigen Jahren durch einen privaten Betreiber gemanagt. Dieser private Betreiber besteht aus einem Konsortium, das durch ein in Großbritannien ansässiges multinationales Wasserunternehmen dominiert wird. Das Wasserunternehmen im Westen, in dem sich unter anderem der zentrale Hafen des Landes befindet, wird als staatliches Unternehmen gemanagt. Das Unternehmen ist zwar im Staatsbesitz, aber die Struktur seines Aufsichtsrats stellt eine hohe Autonomie des Managements sicher. Das dritte Wasserunternehmen im Osten des Landes versorgt vorrangig mittelgroße Städte im ländlichen Raum. Es sollte ursprünglich nach dem Vorbild des Unternehmens im Süden in ein privates Betreibermodell überführt werden. Nach dem Scheitern dieses Privatisierungsmodells wurde angestrebt, dass Unternehmen ökonomisch so leistungsfähig zu machen, dass es als staatliches Unternehmen gemanagt werden kann.

Im folgenden Abschnitt wird herausgearbeitet, wie sich in Bezug auf die einfache Kennzahl des Deckungsbeitrages zwei unterschiedliche Berechnungsformen ausgebildet haben. Im dritten Abschnitt werden drei Machtspiele zwischen internationalen Entwicklungshilfeorganisationen, Ministerien, Behörden und Wasserversorgungsunternehmen rekonstruiert, die sich um den Deckungsbeitrag herum etablieren. Es wird gezeigt, wie eine multinationale Entwicklungsbank vergeblich versucht, die Machtspiele durch ein ITVorhaben zu seinen Gunsten aufzulösen. Im vierten Abschnitt wird herausgearbeitet, weswegen der Deckungsbeitrag trotz der teilweise blockierenden Machtkämpfe im Wassersektor des afrikanischen Landes, eine wichtige Funktion erfüllt: erst durch den Verweis auf den Deckungsbeitrag wird eine Ebene geschaffen, auf deren Basis die Kooperationsbeziehungen immer weiter fortgeführt werden können. Im fünften abschließenden Abschnitt wird versucht herauszuarbeiten, inwiefern sich Zahlenspiele in Entwicklungsländern von Zahlenspielen in Industrieländern unterscheiden.

\section{Die Objektivitätsannahme - Zahlen als Verständigungsmittel}

In den Kooperationsbeziehungen im Rahmen der Entwicklungshilfe spielen verschiedene ökonomische Kennziffern eine Rolle: Anlagevermögen, Anschaffungsvermögen, Cash-Flow, Deckungsrückstellungen, Eigenkapitalquoten, Einnahmen, Erträge, Fremdkapital, Firmenwert, Investitionsrechnungen, Liquiditätspläne, Mankos, Umlaufvermögen, Verbindlichkeiten oder Wiederbeschaffungskosten. In dem untersuchten Wassersektor des afrikanischen Landes ist jedoch eine Kennzahl von besonderer Bedeutung: der Deckungsbeitrag. Der Deckungsbeitrag umfasst das Verhältnis von Betriebseinnahmen zu Betriebsausgaben,

internationalen Beratungsfirmen geführt (sechs Gesprächspartner). Mit über einem Drittel der Gesprächspartner wurden dabei mehrere Interviews geführt, um einen Abgleich mit den Ergebnissen aus anderen Interviews zu ermöglichen. Zusätzlich wurden in der zentralen Wasserbehörde und in einer regionalen Wasserversorgungseinheit teilnehmende Beobachtungen vorrangig in der Finanzbuchhaltung und in der ITAbteilung durchgeführt. Weiterhin wurden zentrale öffentliche und nichtöffentliche Dokumente der Entwicklungshilfeorganisationen, des Ministeriums, der Wasserbehörde und der regionalen Versorger herangezogen. Aus Gründen der Anonymisierung wurden Details betreffend des Landes, der beteiligten Entwicklungshilfeorganisationen und der nationalen Organisationen verändert und auf die Wiedergabe wörtlicher Zitate verzichtet. 
rechnet aber die Kosten, die durch Steuern, Zinsbelastung oder durch Rücklagen für Investitionen entstehen, nicht mit ein. Ein Deckungsbeitrag, der bei $80 \%$ liegt, bedeutet beispielsweise, dass durch die Einnahmen aus Gebühren für das Wasser und dem Verlegen von Anschlüssen die Ausgaben für Personal, Ersatzteile, Büros oder Fahrzeuge nur zu 4/5 gedeckt werden können. Ein Deckungsbeitrag von 150\% bedeutet, dass dem Unternehmen nach dem Abzug der Betriebsausgaben von den Betriebseinnahmen noch $50 \%$ übrig bleiben, um Kredite für Investitionen zurückzuzahlen.

Der Deckungsbeitrag (operating ratio) lässt sich folglich durch ein einfaches Verfahren bestimmen: Es handelt sich um das Verhältnis zwischen den Einnahmen (operating revenues) und den Betriebsausgaben (operating and maintenance costs). ${ }^{8}$ Die Einnahmen sind im Fall der Wasserunternehmen vorrangig die Gebühren, die von den Kunden für die Zurverfügungstellung des Wassers und das Verlegen der Anschlussleitungen verlangt wird. Die Betriebsausgaben sind die Kosten, die den Wasserbehörden für Löhne, Energie und Verbrauchsgüter entstehen. ${ }^{9}$ Sind die Einnahmen niedriger als die Betriebsausgaben liegt der Deckungsbeitrag unter 100\%, Dies ist gerade bei der östlichen Wasserbehörde der Fall, in dem die Betriebskosten aufgrund des bergigen Geländes und dem hohen Anteil von Privatanschlüssen verhältnismäßig hoch sind. Sind die Einnahmen höher als die Betriebsausgaben liegt der Deckungsbeitrag über $100 \%$. Dies wird bisher vor allem in der westlichen Wasserbehörde erreicht. Dies hängt überwiegend damit zusammen, dass im Westen ein vergleichsweise großer Anteil gewerblicher Kunden existiert, die dem Versorger hohe und gut zu kalkulierende Betriebseinnahmen bescheren.

Der Deckungsbeitrag ist in vielen privatwirtschaftlich ausgerichteten Unternehmen nur eine zweitrangige Kennziffer. ${ }^{10}$ Interessieren tun sich Unternehmen vorrangig dafür, ob auch nach dem Bezahlen von Betriebsausgaben, von Zinsen für Kredite, der Bildung von Rücklagen und der Zahlung von Steuern immer noch über 100\% liegt. Aber sowohl nationale Regierung als auch die Entwicklungshilfeorganisationen gehen davon aus, dass die Wasserversorgungsunternehmen des afrikanischen Landes in absehbarer Zeit nicht in der Lage sein werden, aus den Betriebseinnahmen auch die Kreditrückzahlungen, Rücklagenbildung und Steuern zahlen zu können. Ziel, so die zwischen Entwicklungshilfeorganisatio-

8 In der Literatur wird der Deckungsbeitrag häufig nicht als eine Prozentzahl angeben, sondern als absolute Zahl. Dabei wird vom Umsatz die durch die Herstellung verursachten Kosten abgezogen. So entsteht eine Euro- oder Dollar-Summe. Ist diese Summe positiv, leistet das entsprechend fokussierte Produkt einen positiven Beitrag zum Betriebsergebnis. Hier wird unter Deckungsbeitrag jedoch das prozentuale Verhältnis von Betriebseinnahmen zu Betriebsausgaben verstanden.

9 Es handelt sich hier um eine vereinfachte Darstellung auf der Basis der im afrikanischen Land verwendeten Definitionen. In der Literatur wird der Operating Ratio häufig auch umgekehrt bestimmt - als das Verhältnis von Betriebsausgaben zu Betriebseinnahmen. In diesem Fall weist eine niedrige Prozentzahl auf gute, eine hohe Prozentzahl auf schlechte Profitabilitätschancen hin. Diese unterschiedliche Bestimmungsform lässt sich dadurch erklären, dass der Begriff Operating Ratio es offen lässt, wie genau Betriebsausgaben und Betriebseinnahmen zueinander in Verhältnis gesetzt werden.

10 Aus der Perspektive der Accounting-Forschung ist es interessant, wie sich in spezifischen Konstellationen einzelne Kennziffern durchsetzen. Anne Loft hat beispielsweise überzeugend herausgearbeitet, wie sich die Kennziffer der Kostendeckung im Ersten Weltkrieg durchsetzte. Um die Kriegsmaschinerie effizient zu versorgen wurden im Weltkrieg Marktprozesse weitgehend außer Kraft gesetzt und Unternehmen vereinbarte Fixpreise gezahlt. Gerade unter Gewerkschaftern herrschte die Sorge, dass das Kapital versuchen werde ihre Profitrate zu erhöhen, während die Gewerkschaften aufgrund der Kriegssituation zum Lohnverzicht angehalten werden würden. In diesem Klima gewann die Kennzahl der Kostenrechnung an Bedeutung. Sie sollte helfen die ,realen Produktionskosten" zu bestimmen, um so sicher zu stellen, dass die Unternehmenseigner sich neben der Kostendeckung nur einen kleinen Profit genehmigen konnten. 
nen und Wasserministerium vereinbarte Zielsetzung, müsste es jedoch sein, dass die Wasserbehörden wenigstens einen positiven Deckungsbeitrag erzielen: ihre regelmäßigen $\mathrm{Be}-$ triebsausgaben also aus den Einnahmen gedeckt werden können. Der Deckungsbeitrag erscheint deswegen als eine viel wichtigere Kennziffer als die Profitrate. Dementsprechend wird in dem afrikanischen Land über diese Kennziffer versucht, die mit Wasserver- und entsorgung betrauten Organisationen zu steuern. So wird die Auszahlung der Entwicklungshilfe für das für Wasser zuständige Ministerium an eine Verbesserung des Deckungsbeitrages geknüpft. Auch die Möglichkeiten für die regionale Wasserbehörden zu einem staatlichen Unternehmen zu werden und so höhere Autonomie gegenüber Ministerium und Wasserbehörde zu erreichen, ist an einen Deckungsbeitrag über $100 \%$ geknüpft. Und nicht zuletzt werden sowohl die Honorierung privater Betreiberunternehmen im Wassersektor und die Honorierung von Beratern in den regionalen Wasserversorgungseinrichtungen von dem Deckungsbeitrag abhängig gemacht.

\section{Jenseits der Objektivität: Die Konkurrenz zweier Berechnungsverfahren}

Im Wassersektor des afrikanischen Landes haben sich interessanter Weise zwei unterschiedliche Berechnungsverfahren ausgebildet. Im expansiven Modell wird im Zweifelsfall eine Ausgabe immer als Kapitalinvestition und nicht als Betriebsausgabe verrechnet. Ausgaben für Rohre, Wasserzähler und andere Materialen werden als Kapitalinvestition gewertet. Lohnkosten werden dann als Investition abgebucht, wenn die so genannten „Vertragsangestellten" in größeren Infrastrukturvorhaben tätig sind. Im expansiven Modell wird auf der Seite der Betriebseinnahmen großzügig verbucht. So wird jeder in Rechnung gestellter Betrag als Einnahme abgebucht und keine Rücklagen für Zahlungsausfälle gebildet. Im restriktiven Modell werden Ausgaben der Wasserbehörde eher zurückhaltend als Investition verrechnet. Rohre beispielsweise werden nur dann als Kapitalinvestition verrechnet, wenn diese für neue Leitungen genutzt werden. Werden Rohre für Reparaturen eingesetzt, werden diese - ebenso wie die Wasserzähler - als Betriebsausgabe verrechnet. Die „Vertragsangestellte" werden in dem restriktiven Modell genauso wie alle anderen Lohnkosten als Betriebsausgabe gerechnet. Auf der Seite der Betriebseinnahmen wird im restriktiven Modell nicht jeder in Rechnung gestellter Betrag komplett als Einnahme verbucht. Vielmehr wird ein kleiner Teil des Rechnungsbetrages nicht als Einnahme eingebucht, weil davon ausgegangen wird, dass ungefähr $1 \%$ des Rechnungsbetrages nicht bezahlt wird.

In dem afrikanischen Land wird das expansive Modell besonders von der nationalen Wasserbehörde verfochten. Diese Behörde ist im Auftrag des Wasserministeriums für die Erstellung der Einnahmen-Ausgaben-Rechnung für den nationalen Wassersektor zuständig und legt über dieses Berechnungsverfahren den nationalweit erzielten Deckungsbeitrag fest. Gestützt wird dieses Berechnungsmodell durch ein Konsortium von Wasserunternehmen, das als privater Lizenznehmer die Wasserversorgung im Norden betreibt. Dabei verwenden aber weder die nationale Wasserbehörde noch die privaten Betreiber den Begriff des „expansiven Modells", weil für sie diese Berechnungsform des Deckungsbeitrages die einzige vertretbare ist. Dieses restriktive Modell wird besonders von den großen internationalen und nationalen Entwicklungshilfeorganisationen propagiert. Weil diese über Beratungsprojekte teilweise direkten Zugriff auf einzelne regionale Wasserbehörden haben, wird dieses restriktive Modell auch in zwei der drei regionalen Wasserbehörden favorisiert. Von Ver- 
tretern der Entwicklungshilfeorganisationen wird die eigene Berechnungsform als „State of the Art" präsentiert und die Berechnungsmethode der nationalen Wasserbehörde als zu expansiv abgelehnt. Wie kommt es trotz der vermeintlichen „Objektivität“ von Berechnungsverfahren zu der Existenz dieser unterschiedlichen Modelle?

\begin{tabular}{|l|l|}
\hline Expansives Modell & Restriktives Modell \\
\hline $\begin{array}{l}\text { Jedes Rohr wird als Kapitalinvestition } \\
\text { verrechnet }\end{array}$ & $\begin{array}{l}\text { Rohre werden entsprechend ihrer Verwen- } \\
\text { dung zugerechnet. Bei neuen Leitungen } \\
\text { handelt es sich um Kapitalinvestitionen, bei } \\
\text { Reparaturen um Betriebsausgaben }\end{array}$ \\
\hline $\begin{array}{l}\text { Alle Wasserzähler werden als } \\
\text { Kapitalinvestition gezählt }\end{array}$ & $\begin{array}{l}\text { Wasserzähler werden als Betriebsausgabe } \\
\text { verrechnet }\end{array}$ \\
\hline $\begin{array}{l}\text { „Vertragsangestellte“ } \\
\text { (Contract Employees) werden als Kapital- } \\
\text { investition gewertet, weil sie im Rahmen } \\
\text { von Projekten beschäftigt werden }\end{array}$ & $\begin{array}{l}\text { "Vertragsangestellte" } \\
\text { (Contract Employees) werden als } \\
\text { Betriebsausgaben verbucht }\end{array}$ \\
\hline $\begin{array}{l}\text { In Rechnung gestellte Beiträge werden } \\
\text { komplett als Betriebseinnahmen gebucht }\end{array}$ & $\begin{array}{l}\text { Es wird davon ausgegangen, dass } 1 \% \text { alle } \\
\text { in Rechnung gestellten Beträge durch die } \\
\text { Kunden nicht gezahlt werden }\end{array}$ \\
\hline
\end{tabular}

\section{Die Hintergründe zweier unterschiedlicher Berechnungsgrundlagen für den Deckungsbeitrag}

Die Position der Geber ist, dass die nationale Wasserbehörde wenigstens ihre Betriebsausgaben durch ihre Einnahmen decken soll. Das Erreichen dieses Zieles wird als wichtiger Schritt gesehen, damit sich die Wasserbehörden mittelfristig von Zahlungen aus der Entwicklungshilfe unabhängig machen können. Um dieses Ziel zu erreichen, verordnet die Koordinationsrunde der verschiedenen Geberinstitutionen mit der nationalen Wasserbehörde konkrete Ziele: Im nächsten Jahr soll die Wasserbehörde einen Deckungsbeitrag von $130 \%$ erreichen, drei Jahre später von $140 \%$ und fünf Jahre später $150 \%$. Bei Nichterreichung dieser Zielvorgaben drohen die Geber mit einer Reduzierung der Entwicklungshilfezahlungen an das Land. Zur Bestimmung des Deckungsbeitrages sollen, so die Position der Geber, „vernünftige" Berechnungsgrundlagen gewählt werden. So verweisen die Geber immer wieder darauf, dass zur Berechnung des Deckungsbeitrages auf die Bestimmungen der International Financial Reporting Standards und zurückgegriffen werden sollte. Man geht von der Annahme aus, dass nur dieser die „objektive Lage“ einer Behörde und eines Unternehmens abbilden kann und durch diesen Zweifelfragen bei der Zurechnung auf Betriebskosten oder Investitionskosten einwandfrei geklärt werden können. Dabei wird suggeriert, dass es sich bei den International Financial Reporting Standards genauso wie bei seinem Vorläufer, dem International Accounting Standard, um einen einheitlichen Standard und nicht um einen ganzen Apparat von Standards handelt.

Die nationale Wasserbehörde muss sich - nicht zuletzt aufgrund des Drucks des Wasser- und des Planungsministeriums den Vorgaben zum Deckungsbeitrag - beugen. Wenn die Geber „130\% + x Deckungsbeitrag“ wollen, so die Einstellung in der Wasserbehörde, dann werden wir dies auch erreichen. Für sie ist dies eine notwendige Pflichtübung, um 
weiterhin an die Mittel der nationalen und internationalen Entwicklungshilfeorganisationen zu kommen. Anders als von den Gebern intendiert, versucht die nationale Wasserbehörde den Deckungsbeitrag nicht vorrangig durch die Steigerung der Einnahmen oder durch Senkung der Kosten zu erreichen, sondern durch eine Umstellung in der Buchhaltung. Durch die Deklarierung von immer mehr Kosten als Investitionen und durch den Verzicht ausstehende Einnahmen abzuschreiben, wird der Deckungsbeitrag immer weiter erhöht. Dabei besteht die Hoffnung, dass die Geber sich schon nicht allzu intensiv mit den Details der Buchführung auseinandersetzen werden. ${ }^{11}$ Für die Durchsetzung dieses expansiven Modells der Deckungsbeitragsberechnung ist die nationale Wasserbehörde darauf angewiesen, dass die regionalen Wasserbehörden ihren Deckungsbeitrag auch nach dem expansiven Modell berechnen. Bei dem durch die internationale Wasserunternehmen betriebenen Versorger im Zentrum des Landes besteht eine Bereitschaft, dem expansiven Modell zu folgen - hängt ihr Honorar doch teilweise von einem möglichst hohen Deckungsbeitrag ab. Für die regionale Wasserbehörde im Norden ist die Höhe des Deckungsbeitrags nicht von der gleichen Wichtigkeit. Deswegen können auch die von einer europäischen Entwicklungshilfeorganisation installierten Berater, recht erfolgreich diese regionale Wasserbehörde als Modellfall für eine restriktive Deckungsbeitragsberechnung aufbauen. Wie wirken sich jetzt die unterschiedlichen Berechnungsformeln in den Kooperationsverhältnissen in dem afrikanischen Wassersektor aus?

\section{Die mikropolitischen Auseinandersetzungen über die Bestimmung des Deckungsbeitrages}

Im Ansatz des finanziellen Realismus, zu dem neben der betriebswirtschaftlichen Controllingtheorie - auch mit Abstrichen die Tranksaktionskosten- und die Prinicipal-AgentTheorie gezählt werden kann, wird davon ausgegangen, dass die Ermittlung von ökonomischen Kennziffern Machtkämpfe reduzieren können. ${ }^{12}$ Eine Gewinn-Verlust-Rechnung könne dazu beitragen, die Leistungsfähigkeit des Top-Managements einzuschätzen und so deren Bewertung objektivieren. Eine Investitionsrechnung könne eine von politischen Interessen „gereinigte“ Einschätzung einer geplanten Großanschaffung ermöglichen und so die mikropolitischen Spiele reduzieren. Verrechnungspreise zwischen den einzelnen Profitcentern eines Unternehmens könnten sicherstellen, dass die Kooperationsprozesse durch „Marktprozesse" objektiviert werden und so machtpolitische Interessen an Einfluss verlieren.

In dieser Auffassung muss jedoch immer davon ausgegangen werden, dass die Zahlen sich unabhängig von den Interessen beteiligter Organisationseinheiten oder Organisationen bestimmen lassen. Schließlich kann aus der Perspektive des finanziellen Realismus die Reduzierung der Machtkämpfe nur dadurch erreicht werden, dass es eine jenseits der Interessen gelagerte Objektivität gibt, die nicht oder nur mit Schwierigkeiten manipuliert werden können. Diese Einschätzung wird interessanterweise auch in Teilen der gegen den finanziellen Realismus gerichteten kritischen Accounting-Forschung geteilt (konzeptionell

11 Aus Fallstudien über Unternehmen in Industrieländern wissen wir, wie durch kreative Buchführung punktgenau von oben gesetzte Ziele erreicht werden (vgl. z.B. eindrucksvoll Jackall 1988: 107ff).

12 In der Literatur wird dieser Ansatz auch als ,positive Accounting-Forschung " oder in Abgrenzung von den "critical accounting theory studies" (cats) auch als ,rational accounting theory studies" (rats) bezeichnet. Für gute Theorieüberblicke siehe z.B. Chua 1986: 601ff; Becker 2003: 61ff. Einen guten kurzen Überblick liefert auch Power 1993. 
siehe Burchell et al. 1980; Hopwood 1987). Aus einer marxistischen Perspektive beschreiben Tinker, Lehman und Neimark Accounting als eines der letzten ,nicht politisierten Mysterien" (Tinker et al. 1988: 213). Aber auch in der sich auf Michel Foucault beziehenden Accounting-Forschung wird argumentiert, dass Zahlen häufig nicht mehr "aufgefaltet" werden können und subtile Machtregulierungsmechanismen einsetzen (vgl. Hacking 1990; Rose 1991). Der Unterschied zwischen dem finanziellen Realismus und der an Marx und Foucault anknüpfenden Accounting-Forschung ist lediglich, dass die Anhänger einer Marxschen oder Foucaultschen Argumentation hinter diesen konfliktreduzierenden Mechanismen kaschierte Machtinstanzen vermuten. Die Konstruktion von Zahlen werde durch Experten bewacht, die darüber zentrale Prozesse in Organisationen beherrschen. ${ }^{13}$

Im Fall des Wassersektors in dem afrikanischen Land wird jedoch deutlich, wie schwierig dieser Schutzprozess ist. Zwischen den Gebern auf der einen Seite und der Wasserbehörde auf der anderen Seite herrscht seit knapp einem Jahrzehnt ein immer komplexer werdendes Machtspiel: Die Geber insistieren, dass die Wasserbehörde einen vorher definierten Deckungsbeitrag erreichen soll und legen dies in "Aktionsplänen“ für die Wasserbehörde vor. Die Wasserbehörde reagiert auf diese Anforderung mit immer stärker aufgeweichten Systemen zur Bestimmung des Deckungsbeitrages und erreicht so punktgenau die in den "Aktionsplänen" festgelegten Beträge. Die Geber wiederum versuchen die Aufweichung dieser Standards zu verhindern, um ihre eigentliche Intention - eine Effizienzsteigerung der Wasserbehörde - zu erreichen. Vor dem Hintergrund dieses grundlegenden Machtspiels laufen dann verschiedene andere Machtspiele ab, die maßgeblich den Wassersektor in dem afrikanischen Land prägen.

\section{Die Machtspiele um den Deckungsbeitrag}

Bei der Auseinandersetzung um den Deckungsbeitrag stehen sich in der Regel immer die nationale Wasserbehörde auf der einen Seite und die Gruppe der Geberorganisationen auf der anderen Seite gegenüber. Die Position der anderen Organisationen hängt davon $a b$, welche Berechnungsmethoden für sie jeweils vorteilhafter sind. Dabei bilden sich Interessenskonstellationen aus, die quer zu einer Konfliktlinie zwischen den Organisationen aus der nördlichen Halbkugel einerseits und den Organisationen im Entwicklungsland andererseits liegen. Ein erstes Machtspiel dreht sich dabei um die Entlohnung der über die Entwicklungshilfe bezahlten privaten Betreiber. Dabei bildet sich eine Koalition zwischen der zentralen Wasserbehörde und dem privaten Betreiber im Norden des Landes aus. Dieser privaten Betreiber, an dem neben dem internationalen Wasserunternehmen auch ein internationales und ein nationales Beratungsunternehmen beteiligt ist, hat die Wasserwerke und Versorgungs- und Entsorgungsleitungen nicht erworben, sondern erhält für den Betrieb des Systems ein erfolgsabhängiges Honorar. Das Honorar dieser Beraterfirma hängt maßgeblich davon $a b$, in welchem Maße das Wasserunternehmen seinen Deckungsbeitrag steigern kann. Erst bei einer deutlichen Steigerung des Deckungsbeitrages wird das private Betreibermodell für das internationale Versorgungsunternehmen und die beiden Beratungsunternehmen finanziell interessant. Aus diesem Grund unterstützt das private Konsortium, die

13 Machtprozesse sind in der Accounting-Forschung lange Zeit vorrangig für Budgetierungsprozesse untersucht wurden (vgl. z.B. Argyris 1952; Wildavsky 1964; Wildavsky 1975; Covaleski/Dirsmith 1986; speziell für Budgetierung in Entwicklungsländern Caiden/Wildavsky 1974). 
expansive Berechnungsmethode der zentralen Wasserbehörde, kommt dieses expansive Modell doch immer zu höheren Deckungsbeiträgen.

Die Geber versuchen diesem Effekt über den Aufsichtsrat des Wasserunternehmens im Zentrum entgegenzusteuern, um die Erosion ihrer Berechnungsstandards zu verhindern. Sie nutzen dafür teilweise auch die von ihnen eingerichtete Project Management Unit in der zentralen Wasserbehörde, über die die privaten Betreiber kontrolliert werden sollen. Ergebnis ist eine permanente Auseinandersetzung zwischen Aufsichtsorgan, der Project Management Unit und dem privaten Wasserunternehmen über Details des Abrechnungsverfahrens. Diese Konfliktlinie in dem afrikanischen Land lässt sich bei vielen in Amerika, Asien und Afrika etablierten privaten Betreibermodelle finden. Die Ausgangssituation („Baseline") für die Leistungsbemessung ist - trotz teilweise hohen Messaufwand zu Beginn des Vertrages - nicht eindeutig zu bestimmen. Das betrifft sowohl die in die Landeswährung gefassten als auch die nichtmonetären Kennzahlen. Aus verschiedenen Fallstudien besonders über Privatisierung in der Wasserversorgung ist bekannt, dass auch bei privaten Betreibermodellen, in denen die Entlohnung an die Anzahl von neu verlegten Wasseranschlüssen, an die Verringerung von Wasserverlusten oder an die Reduzierung von Betriebskosten gebunden werden, es zu heftigen Auseinandersetzungen über die Interpretation dieser Zahlen kommt (vgl. Megginson/Netter 2001: 346; Azpiazu/Focinito 2002; Grusky 2003; Ugaz 2002). ${ }^{14}$

Das zweite Machtspiel findet zwischen der nationalen Wasserbehörde und den regionalen Wasserunternehmen statt. Über größere Beratungsprojekte haben die Geber direkten Zugriff auf die regionalen Wasserversorger im Westen und im Osten des Landes. Über diese Beratungsprojekte versuchen die Geber ihr restriktiveres Berechnungssystem für den Deckungsbeitrag durchzusetzen. Besonders im Osten versuchen die Berater in Abstimmung mit den europäischen Gebern das Buchhaltungssystem als Modell für das ganze Land aufzuarbeiten. Die regionalen Wasserversorger sind jedoch nach wie vor der zentralen Wasserbehörde unterstellt. Der Geschäftsführer der regionalen Wasserbehörde ist hierarchisch dem Behördenchef der nationalen Wasserbehörde zugeordnet und auch indirekt noch von Mittelzuweisungen durch die zentrale Wasserbehörde abhängig. So kann die zentrale Wasserbehörde durchsetzen, dass die regionalen Wasserbehörden an die nationalen Standards „angepasste Betriebsergebnisse" produzieren. Der Effekt ist, dass zwei Betriebsergebnisse mit jeweils unterschiedlichen Deckungsbeiträgen existieren. Im Osten wird dann einerseits mit dem restriktiven Berechnungssystem der Geber ein Deckungsbeitrag von $60 \%$ errechnet, während mit dem expansiven Berechnungssystem der nationalen Wasserbehörde ein Deckungsbeitrag von $70 \%$ erzielt wird.

Das dritte Machtspiel dreht sich um die Überführung der regionalen Wasserbehörden in staatliche Unternehmen. Der Deckungsbeitrag ist unter anderem deswegen eine kritische Kennziffer, weil die - besonders von den Gebern geforderte - Umstrukturierung der regionalen Wasserbehörden in staatliche Unternehmen von einem Deckungsbeitrag von über $100 \%$ abhängig gemacht wird. Hintergrund ist ein nationales Gesetz, wonach staatliche Unternehmen nur gegründet werden dürfen, wenn diese mittelfristig ökonomisch lebensfähig sind. Gerade bei der Verhandlung über die Überführung des regionalen Wasserbehörde im Norden in ein staatliche Unternehmen entsteht eine fast paradox wirkende Situation: Die

14 Für das eine der beiden private Betreibermodell in Manila wurde gezeigt, wie trotz intensiver Bemühungen zur Definition der Ausgangssituation es zu unterschiedlichsten Interpretationen des Vertragswerks kam (vgl. Stadler/Hoering 2003; 150ff). 
Geber, die auf eine möglichst schnelle Umwandlung der Wasserbehörde dringen, sehen sich an ihre restriktive Berechnungsformel gebunden. Auch wenn sie für eine möglichst schnelle Überführung in einen neuen Rechtsstatus sind, erreicht die betroffene Wasserbehörde nach ihrer Berechnungsform wesentlich später den notwendigen Deckungsbeitrag von $100 \%$ als nach der expansiven Berechnungsformel der nationalen Wasserbehörde. Die zentrale Wasserbehörde, die einer rechtlichen Unabhängigkeit eines lokalen Ablegers eher zögerlich gegenüber steht, kommt mit ihren expansiven Berechnungen zu einem eher schnellen Erreichen des notwendigen Deckungsbeitrages von $100 \%$. Hierbei wird deutlich, dass Organisationen mit widersprüchlichen organisationalen Zielsetzungen konfrontiert sein können (vgl. hierzu aus unterschiedlichen Theorieperspektiven Luhmann 1973; Friedland/Aldorf 1991). Die Zielvorstellungen der Entwicklungshilfeorganisation, die regionalen Wasserversorger möglichst schnell aus den Fängen der nationalen Wasserbehörde zu befreien, stehen im Konflikt mit dem Ziel einer möglichst restriktiven Buchführung. Die Zielvorstellung der nationalen Wasserbehörde eine Autonomie der regionalen Wasserversorger möglichst weit herauszuzögern gerät im Widerspruch zu der expansiven Bestimmung des Deckungsbeitrages.

In allen dieser drei Machtspiele ist auffällig, wie stark sich die Akteure an ihre jeweiligen Berechnungssysteme gebunden sehen. In dem Konflikt über die Leistungsfähigkeit wird tendenziell sowohl von der Wasserbehörde als auch von den Entwicklungshilfeorganisationen immer mehr aus dem Auge verloren, dass der Deckungsbeitrag keine Aussage über die ökonomische Überlebensfähigkeit eines Unternehmens bietet. Um diese zu bestimmen, wäre es notwendig für die betriebswirtschaftliche Rechnung auf der Kostenseite auch Ausgaben für Steuem, Zinsen und Rücklagen für Investitionen mit einzukalkulieren.

\section{Die technische Lösung für ein mikropolitisches Problem}

Nachdem der Konflikt über den Deckungsbeitrag die Auseinandersetzung im Wassersektor des afrikanischen Landes über längere Zeit geprägt hat, gab es gerade von Seiten der Geber den Versuch, einen einheitlichen Accounting Standard für das Land zu etablieren. Besonders eine multilaterale Entwicklungsbank verlangt von der zentralen Wasserbehörde ein technisches Projekt aufzulegen, mit dem das Rechnungswesen in dem Land vereinheitlicht werden soll. Gerade für die Politik der Weltbank ist inzwischen gut herausgearbeitet worden, wie diese Finanz- und Accounting-Technologien auch an weit entfernte Plätze transportiert werden. Dabei sind Projektdesigns, Kreditabkommen oder beraterische Unterstützungsmaßnahmen Instrumente, mit denen Vorstellungen der Weltbank von Finanzinstrumenten und Rechnungslegungsstandards transportiert werden (vgl. Neu et al. 2002; Neu/Ocampo 2006; siehe auch Harper 1998). ${ }^{15}$ Zur Durchsetzung dieser Vereinheitlichung finanziert - inspiriert durch die multilaterale Entwicklungsbank - eine große amerikanische Entwicklungsagentur ein über fünf Jahre laufendes Projekt zur Einführung eines „Finanziellen Berechungssystems" (FBS), Offizielles Ziel dieses Projektes ist es, das alte budgetbasierte Buchfuhrungssystem auf ein moderneres und flexibleres Berechnungsverfahren umzustellen und landesweit die Einnahme- und Ausgabeverbuchung auf EDV umzustellen. Die versteckte Agenda

15 Bei Jang (2006) findet sich aus einer neoinstitutionalistischen Perspektive die Argumentation, dass Accounting stark in die „Weltkultur" eingebettet sei. Es gebe, so Jang, einen weltweiten Anstieg von Organisationen, die sich den Accounting-Normen unterwerfen. 
bei der Vereinheitlichung des Accounting Standards ist jedoch, über ein landesweites System das von den Gebern propagierte restriktivere Berechnungssystem durchzusetzen. Die Hoffnung ist, dass durch die Abhängigkeit der Software-Entwickler von den Zahlungen der Geber, Buchungsverfahren zugrunde liegen, die eher zurückhaltend mit der Einbuchung von Kosten als Investitionen sind.

James Ferguson hat in einer Studie über Weltbank in Lesotho die Strategie von multilateralen Entwicklungshilfeorganisationen als "Anti-Politics-Machine" bezeichnet. Die Entwicklungshilfeorganisationen würden zentrale Entwicklungsprobleme wie zum Beispiel Armut auf "technische Probleme" reduzieren. Für diese technischen Probleme würden die Entwicklungshilfeorganisationen dann auch rein technische Lösungen suchen. Dies trüge zu einer Entpolitisierung der Entwicklungshilfe bei und würde, so Ferguson, von den wirklichen Problemen in Entwicklungsländern ablenken (vgl. Ferguson 1990, 1994). ${ }^{16}$ Ferguson bezieht sein Argument vorrangig auf die Abstinenz von Entwicklungshilfeorganisationen von der ,großen Politik“. ${ }^{17}$ Seit Ende des Kalten Krieges hat sich die Rhetorik der Entwicklungshilfeorganisationen jedoch grundlegend verändert. An der Karriere des Themas Korruption kann man erkennen, dass selbst die Weltbank - früher sicherlich der Prototyp einer Anti-Politics-Machine - bewusst politische Eckpfeiler für Länder festlegt. Bei den nationalen Entwicklungshilfeorganisationen findet sich inzwischen häufig das Selbstverständnis einer „politischen Entwicklungszusammenarbeit“.

Das Bild der „Anti-Politics-Machine“ lässt sich jedoch sinnvoll beibehalten, wenn man es nicht auf die Rhetorik der Entwicklungshilfeorganisationen bezieht, sondern auf die Vielzahl von Programmen, Projekten und Maßnahmen, die von den Organisationen durchgeführt werden. In diesen Prozessen der „Projektifizierung“, der „Programmierung“ und „Maßnahmendefinition" werden die mikropolitischen Komponenten „verobjektiviert". Die Projekte, Programme und Maßnahmen erscheinen nur noch als technische Maßnahmen, von ihren politischen Komponenten vollkommen beraubt. Das Finanzielle Berechnungssystem (FBS) erscheint als ein rein technisches Projekt. Das Ausgangsproblem, an dem das Projekt ansetzt, wird rein technisch definiert, indem auf die veraltete IT-Architektur der Wasserbehörde verwiesen wird. Als Lösung wird dann auch die Schaffung eines neuen ITSystems angesehen, in dem sowohl die Einnahmen und Ausgaben zeitnah und behördeneinheitlich verbucht werden können. Als Experten für das Problem werden dann folglich auch SAP-Spezialisten und Datenbank-Programmierer angesehen. Durch diese technische Projektanlage wird der Interessenskonflikt zwischen Gebern und Wasserbehörde durch die „Anti-Politics-Machine" entpolitisiert.

Aber die Prozedur der "Anti-Politics-Machine“ fuihrt - jedenfalls in dem Fall des afrikanischen Staates - nicht dazu, dass die Machtauseinandersetzungen aufgelöst werden können. Gerade für die zentrale Wasserbehörde ist die versteckte Agenda hinter dem Software-Projekt leicht zu erkennen. Obwohl eine neue Software auch aus der Wahrnehmung der zentralen Wasserbehörde dringend benötigt wird, blockiert diese das Software-Projekt, immer wenn es um die Zuordnung von Ausgaben in die Bereiche Betriebskosten und Investitionskosten geht. Effekt dieser technischen Lösung für das ursächlich mikropolitische

16 Das generalisierte Argument von Ferguson findet sich schon bei Meltsner (1976: 32). Die Studie von Ferguson ist in den späten achtziger, frühen neunziger Jahren entstanden und betrifft besonders die Situation multilaterale Entwicklungsbanken. Diese scheine aufgrund ihrer komplexen Besitzverhältnisse stärker als die bilateralen Entwicklungsbanken auf politische Zurückhaltung angewiesen zu sein. 
Problem war, dass sich das Software-Projekt um Jahre verspätete und es sich andeutete, dass am Ende lediglich eine Software-Ruine von den Beratern übergeben wird.

\section{Die Verschärfung der mikropolitischen Spiele durch die Orientierung am Deckungsbeitrag}

Schon Aaron Wildavsky (1964) hat in seinem "The Politics of the Budgetary Process" Zweifel angemeldet, dass sich mit Kosten-Nutzen-Analysen, mit Programmbudget oder mit ökonomischen Kennziffernsystem der politische Bargaining Prozess reduzieren lässt. Aufgrund der hier vorgestellten Fallstudie lässt sich Wildavskys Argument noch verschärften: Die Versuche, mikropolitische Prozesse durch ökonomische Kennziffersysteme zu reduzieren, führt eher zu einer Vervielfältigung der Konflikte. Man kann anhand der Fallstudie geradezu eine mikropolitische Paradoxie des Accountings in der Entwicklungshilfe feststellen. Die „Zahlensteuerer" verlängern und verkomplizieren die mikropolitischen Prozesse, die sie eigentlich reduzieren wollen. Ausgefeilten Kennzahlen-Systemen raffinieren, was sie eigentlich beseitigen sollen. Kennzahlen können letztlich langfristig die Konflikte eskalieren lassen, die durch den Einsatz der Kennzahlen eigentlich reduziert werden sollen (vgl. Neuberger 2001: 192).

\section{Die Funktion von ökonomischen Kennziffern in der Entwicklungshilfe}

Die Verkomplizierung der Machtspiele durch die Orientierung aller Beteiligten an einer Verbesserung des Deckungsbeitrages erscheint auf den ersten Blick wie eine weitere $\mathrm{Ne}$ benfolge in der Entwicklungshilfe. Genauso wie ein gut gewolltes integriertes Landwirtschaftsprojekt zu einer Überweidung beitragen kann oder die Entwicklungshilfe für einen Staat dazu führt, dass sich korrupte Eliten an der Macht halten, könnte auch die Orientierung am Deckungsbeitrag mit einem ,gut gewollt, aber problematisch in seinen Folgen“" abgehakt werden. Aus dieser Perspektive könnte die Orientierung am Deckungsbeitrag als ein vorrangig durch eine in dem Wassersektor dominierende multilaterale Entwicklungsbank zu verantwortender "Steuerungsfehler" interpretiert werden. So setzt bei den Entwicklungshilfeorganisationen und bei den in den verschiedenen Projekten aktiven Beratungsunternehmen in den letzten Jahren zunehmend Kritik an der Orientierung an einer Erhöhung des Deckungsbeitrages ein. Aus einer distanzierten soziologischen Perspektive scheint es jedoch sinnvoll diese schnelle Kritik in Frage zu stellen. Selbst wenn die offensichtlichen, manifesten Funktionen der Deckungsbeitragsorientierung nicht erreicht werden, so können doch eventuell versteckte, latente Funktionen beim Deckungsbeitrag eine Rolle spielen. Das von Robert Merton eingeführte Denkkonzept der latenten Funktion ermöglicht es, sich von den Selbstbeschreibungen von Familien, Stämmen, Organisationen oder Gesellschaften zu lösen und Funktionen aus einer distanzierteren Perspektive heraus zu bestimmen (vgl. Merton 1952). Schließlich umfasst eine Familie, ein Stamm, eine Organisation oder eine Gesellschaft immer mehr als sie prima facie darstellen (vgl. Luhmann 1967). 


\section{Die kurzfristige Entlastung der Verhandlungssituation durch die Orientierung am Deckungsbeitrag}

Eine zentrale Funktion des Deckungsbeitrages scheint darin zu bestehen, dass in kritischen Verhandlungssituationen wenigstens kurzfristig eine Grundlage geschaffen wird, auf die sich - bei aller Widersprüchlichkeit - die beteiligten Organisationen einigen können. Der Effekt von ökonomischen Kennziffern scheint zu sein, dass durch den Verweis auf eine gemeinsame „Sprache“ ein Konflikt kurzfristig befriedet wird (vgl. auch Porter 1995: ix). Man einigt sich in den Aushandlungen zwischen den Gebern und der Wasserbehörde daraus, dass die Leistungen zukünftig am Erreichen von Deckungsbeiträgen gemessen werden soll. Oder in den Verhandlungen zwischen Wasserbehörde, Geberorganisationen und privaten Betreibern wird die Honorarzahlung an die Verbesserung von Deckungsbeiträgen geknüpft. Auch wenn diese Befriedung nur kurzfristig wirkt, weil sich die Machtspiele dann in den Projekten, Programmen und Maßnahmen, die über die Kennziffer gesteuert werden multiplizieren: In dem Moment der Einigung auf eine ökonomische Kennziffer gibt es erstmal einen Anlass weiterzumachen. Überspitzt ausgedrückt: Über Zahlen wird die Fiktion einer Einigung produziert, auf dessen Basis die verschiedenen Organisationen weiter miteinander kooperieren können.

Der Clou ist, dass diese Funktion auch erfüllt wird, wenn die Kennzahl nur sehr lose mit der Realsituation gekoppelt ist. Zahlen geben bei aller Konstruiertheit Sicherheit, weil auf deren Basis Entscheidungen gefällt werden können. Die „Willkürlichkeit“ von Zahlen wird - ähnlich wie das "Stoppen bei Rotlicht" - in Kauf genommen, weil sie sich als Grundlage für Entscheidungen eignen. Bei allem Abstand zur Realsituation können Zahlen also durchaus reale Konsequenzen haben (vgl. Macintosh et al. 2000: 13, 42). Damit die Zahlen ihre realen Konsequenzen haben können, müssen sie jedoch ,selbstverständlicht“ werden.

\section{Der Prozess der „Selbstverständlichung “ von Kennzahlen}

Im Fall der afrikanischen Wasserbehörde kann gezeigt werden, wie die ökonomische Kennziffer des Deckungsbeitrages einen immer selbstverständlicheren Charakter bekommt. ${ }^{18}$ In den mündlichen und schriftlichen Verhandlungen zwischen den großen Geberorganisationen, der nationalen Wasserbehörde und den lokalen Wasserversorgern spielen die unterschiedlichen Berechnungsformen noch eine große Rolle. Immer wieder finden Auseinandersetzungen darüber statt, wie genau der Deckungsbeitrag zu bestimmen ist. Für die unmittelbar an den Verhandlungen beteiligten Vertreter der verschiedenen Organisationen liegt die Konstruiertheit dieser Kennziffer noch im Wahrnehmungshorizont. Aber je mehr sich die Kommunikationen von der unmittelbare Verhandlungssituation zwischen den Repräsentanten der Organisationen zu den internen Abstimmungen in den jeweiligen Organisationen verschiebt desto stärker verblasst dieser Konflikt. Das Wasserministerium kann dem Planungsministerium melden, dass der von der Wasserbehörde erwartete Deckungs-

18 Von Wai Fong Chua gibt es eine Beschreibung eines ähnlichen Prozesses in drei australischen Krankenhäusern. Die Etablierung von Accounting-Systemen wird nicht aus einem Wissen über die ökonomische Nützlichkeit erklärt sondern vielmehr als Resultat von ,rhetorischen Strategien " von Experten (vgl. Chua 1995: 111 und 113). 
beitrag punktgenau erreicht wurde. In den Studien zu privaten Betreibermodellen in dem afrikanischen Land wird zwar immer wieder darauf verwiesen, dass die Organisationen wenigstens ihre laufenden Kosten erwirtschaften sollen, aber die Unterschiedlichkeit der Berechnungsmethode wird dabei nicht erwähnt. In den Dokumenten zu den Regierungsverhandlungen wird nur noch von einer Verbesserung des Deckungsbeitrages geredet, ohne dass aber die Konflikte spezifiziert werden.

Trotz des ursprünglichen Konfliktes über die Bestimmung wird die Kennziffer Deckungsbeitrag in der Prozessierung in den jeweiligen Organisationen immer selbstverständlicher. Die Berichte mit den Zahlenwerken werden, so Richard Rottenburg, bei ihren "Wanderung durch die Abteilungen und Hierarchien" immer dünner. Die Informationen werden nach vorgegebenen Transformationsregeln in immer neue Formen umgewandelt und dadurch immer weiter reduziert. Dadurch stehen am Ende des Prozess nur noch einige wenige Kennzahlen, die von ihren Entstehungs- und Kontextbedingungen weitgehend entkleidet sind und so die Notwendigkeit für vertiefte Kenntnisse sozialer Situationen reduziert (vg1. Rottenburg 2002: 223; siehe schon früher Porter 1995: ix).$^{19}$ Die in den jeweiligen Verhandlungssituationen noch offensichtlichen konflikthaften Konstruktionsbedingungen des Deckungsbeitrages verschwinden so in den internen Abstimmungsprozessen der multilateralen Entwicklungsbanken, der nationalen Entwicklungshilfeorganisationen und der Ministerien des afrikanischen Landes. Die komplexe Realität eines Wasserversorgungsvorhabens wird auf eine Zahl wie „Deckungsbeitrag von 105\%“ reduziert. Je allgemeingültiger diese Kennziffer ist desto weniger könnte die „Vielfalt“", „Komplexität" und „Partikularität" einer ,ortsgebundenen Wirklichkeit" berücksichtigt werden (vgl. Rottenburg 2002: 229f). Die Konstruktionsformen des Deckungsbeitrages können in dem immer weiter fortschreitenden Prozessionsprozess nur noch mit größter Mühe aufgeschnürt werden und erhalten so ein hohes $\mathrm{Maß}$ an Plausibilität.

Aus dieser Plausibilisierung beim Prozessieren innerhalb einer Organisation kann auch erklärt werden, weswegen sich Kennziffern wie der Deckungsbeitrag so gut für die Einigungsfiktionen eignen. Auch wenn den unmittelbar an den Verhandlungen Beteiligten klar ist, dass es keine Übereinstimmung über die Details der Bestimmung einer Kennziffer gibt, so können sie durch davon ausgehen, dass die eigene Organisation bei der Behandlung der Einigung von diesen Details sehr wohl abstrahieren wird.

\section{Eigenschaften von Zahlen und der Prozess der ", Selbstverständlichung“}

Die Plausibilisierung findet für alle Formen von Informationen statt, die in einer Organisation kondensiert und komprimiert werden. Auch qualitativ aufbereitete Informationen erhalten durch ihre Verdichtung und Aufbereitung eine größere Überzeugungskraft. Man denke nur an die für Insider überraschend rund wirkenden Vorstandsvorlagen oder die mehrmals überarbeiteten Powerpoint-Präsentationen, die aufgrund ihrer Stringenz kaum noch kritisierbar erscheinen. Zahlen scheinen aber über drei Merkmale zu verfügen, die sie für den Prozess der „Selbstverständlichung“ besonders geeignet erscheinen lassen. In der Form von Kennziffern sind Informationen besonders leicht transportierbar. Weil es sich

19 Insgesamt ist für Rottenburg jedoch die „Liste“ wichtiger als die „Zahl“. Aus meiner Sicht müsste geprüft werden, ob es nicht die durch Listen mögliche Quantifizierung ist, die diese in der Entwicklungshilfe so attraktiv macht. 
um verdichtete Zahlen handelt, können sie zwischen Profitcentern und Zentrale, zwischen Zulieferern und Kunden, zwischen Unternehmen und Steuerbehörden, zwischen Gebern und Nehmern von Entwicklungshilfe hin- und hergeschoben werden. So kann die Kennziffer des Deckungsbeitrags beispielsweise auf der Ebene der drei Wasserver- und Entsorgungseinheiten erhoben und dann zur zentralen Wasserbehörde weitergeleitet werden. Oder der Deckungsbeitrag der nationalen Wasserbehörde kann an die Entwicklungshilfeorganisationen weitergeleitet werden, bei der ein Projektmanager mit einem einfachen Blick erfassen kann, ob die Zielvorgaben erreicht wurden oder nicht.

Wenn die Informationen in Form von Zahlen aufbereitet sind, sind diese auch im Vergleich beispielsweise zu verbalen Informationen relativ leicht kombinierbar. Der Deckungsbeitrag der drei Wasserversorgungseinheiten kann beispielsweise zu einem einheitlichen Deckungsbeitrag der nationalen Wasserbehörde aggregiert werden. Und wenn eine Entwicklungshilfeorganisation Interesse daran hat, könnte sie ohne größere Schwierigkeiten den durchschnittlichen Deckungsbeitrag alle von ihr finanzierten Versorgungsunternehmen berechnen. ${ }^{20}$ Ein weiteres Charakteristikum von Zahlen besteht darin, dass Sie ohne große Schwierigkeiten vergleichbar sind. ${ }^{21}$ Der Deckungsbeitrag von $150 \%$ in der einen Wasserbehörde kann mit dem Deckungsbeitrag von $70 \%$ bei einer anderen Behördeneinheit verglichen werden. Oder der Deckungsbeitrag von 50\% im Jahr 2000 kann mit dem Deckungsbeitrag von $110 \%$ im Jahr 2010 verglichen werden. Während bei qualitativen Informationen regelmäßig der Vorwurf gemacht wird, dass Äpfel mit Birnen verglichen wird, sind die Vergleiche mit der gleichen ökonomischen Kennziffer in der Regel nicht diesem Verdacht aufgesetzt. Die drei Merkmale der Transportierbarkeit, Kombinierbarkeit und Vergleichbarkeit von Zahlen macht deren Einsatz in der Entwicklungshilfe so attraktiv. Erst sie ermöglichen es den nationalen und internationalen Entwicklungshilfeorganisationen Projekte auch auf große Distanz zu steuern und trotz ganz unterschiedlicher regionaler und kultureller Besonderheiten der Einsatzgebiete mit einheitlichen Kriterien arbeiten zu können.

\section{Fazit: Zahlen in der Entwicklungshilfe}

Die Validität von Zahlen werden in sozialen Zusammenhängen unterschiedlich stark abgesichert. Es gibt akzeptierte mathematische Formeln wie das berühmte , $2+2=4$ “, deren Gültigkeit nur von Grundschülern in der ersten Klasse oder besonders raffinierten Philosophen bezweifelt werden kann (vgl. Bloor 1994). Es gibt die Anzahl von verlegten Wasserleitungen in Tansania, über deren Anzahl unterschiedlichste Meinungen existieren mögen, deren Bestand aber im Rahmen eines Projektes objektiviert werden kann (vgl. Rottenburg 2001). Und es gibt Indikatoren wie dem Human-Development-Index der Vereinten Nationen oder den Korruptionsindex von Transparency International, bei dem die „soziale Konstruiert-

20 Auf die Kombination von Mobilität, Kombinierbarkeit und Stabilität bei Zahlen macht in Anschluss an Bruno Latour z.B. Robson (1992: 690f) aufmerksam (siehe auch Latour 1990). Besten Dank an Andrea Mennicken für den Hinweis auf den Latourschen Ansatz.

21 Quantifizierung, so Stefan A. Musto (1987: 428), würde zusammen mit Standardisierung und Kausalisierung den Entwicklungshilfeorganisationen ermöglichen ein ,simples Weltbild“" zu erstellen. Die Quantifizierung mache Vergleiche möglich. Die Standardisierung erlaube Verallgemeinerungen. Die Kausalisierung, die Aufstellung eindeutiger Folgerungen, begründe Handlungsanweisungen. Dies "Vereinfachungen" seien funktional, weil ein ,wirklichkeitsnäheres Weltbild“" gezielte Einzeleingriffe gar nicht möglich erscheinen lassen. 
heit" selbst in den Massenmedien diskutiert wird (vgl. Booysen 2002). Für eine soziologische Analyse ist jetzt interessant, wie eine Objektivierung von Zahlen von statten geht. Viele Rechnungs- und Auditierungsverfahren machen die Kontingenz von Berechnungen unsichtbar und produzieren so Zahlen, die von den unmittelbar Betroffenen und den späteren Verwendern akzeptiert werden. In der Accounting-Forschung werden diese Prozesse mit Begriffen wie „Enactment“ (Radcliffe 1999), „Upkeying“" (Vollmer 2007) oder „MicroProduction of Macro-Order" (Pentland 1993) bezeichnet. Was ist jetzt das Besondere dieser Prozesse des „Enactment“, „Upkeying“ oder „Micro-Production of Macro-Order“ im Kontext der Entwicklungshilfe?

Stark vereinfachend gedacht kann man in Industrieländern davon ausgeben, dass bei der Bestimmung beispielsweise einer Gewinn- und Verlustrechnung oder eines Deckungsbeitrages bewährte Verfahren gibt, in denen im Konfliktfall die ökonomische Kennziffer sozial objektiviert werden können. Wenn der Verdacht besteht, dass das Management vergangene Gewinne eines Unternehmens durch Zahlentricks in die Höhe getrieben hat, kann ein Aktionär vor Gericht ziehen. Wenn die Steuerbehörde eines Landes den Eindruck gewinnt, dass Unternehmen Einnahmen ins Ausland verschieben, können sie im Rahmen des Gesetzes prüfen, ob die Gewinn-Verlust-Rechnung modifiziert werden muss. Die Justiz hat dabei natürlich auch nicht die Möglichkeit, realitätsnähere Berechnungsverfahren zu erstellen. Vielmehr besteht ihre Funktion darin, dass sie Konflikte über Zahlen entscheiden kann. Dabei kann es sehr wohl passieren, dass das durch ein Gerichtsurteil „objektivierte“ Berechnungsverfahren allen anderen Beteiligten abstrus erscheinen, aber die Beteiligten haben eine Basis auf der alle weitermachen können. Die Möglichkeit zur juristischen Eskalation unterschiedlicher Interpretation von Zahlen wirkt wie eine Stoppregel für Konflikte. Diese Möglichkeit besteht in der Entwicklungshilfe nur begrenzt. Konfliktparteien verlassen sich im Kontext der Entwicklungshilfe sehr ungern auf die Justiz, um rechnerischen Sachverhalten klären zu lassen. Gerade die binationalen und multinationalen Entwicklungshilfeorganisationen vertrauen den rechtlichen Instanzen in den Empfängerländern nicht zu, die Interpretationskonflikte zu lösen. Effekt ist, dass die Zahlen - bei allen vorhandenen Dialekten und Interpretationsschwierigkeiten - in der Kooperation zwischen den verschiedenen Organisationen immer wieder bestätigt werden.

\section{Literatur}

Ahrens, T. (1996): Styles of Accountability. In: Accounting, Organizations and Society 21, S. 139-173. Ahrens, T. (1997): Talking Accounting: An Ethnography of Management Knowledge in British and German Brewers. In: Accounting, Organizations and Society 22, S. 617-637.

Ansari, S./Euske, K.J. (1987): Rational, Rationalizing, and Reifying Uses of Accounting Data in Organizations. In: Accounting, Organizations and Society 12, S. 549-570.

Argyris, C. (1952): The Impact of Budgets on People. Ithaca, NY: Cornell University Press.

Azpiazu, D./Focinito, K. (2002): Privatisation of the Water and Sanitation Systems in the Buenos Aires Metropolitian Area: Regulatory Discontinuity, Corporate Non-performance, Extraordinary Profits and Distributional Inequality. Oxford: Prinwass Oxford.

Becker, A. (2003): Controlling als reflexive Steuerung von Organisationen. Stuttgart: Schäffer-Poeschel.

Bloor, D. (1994): What Can the Sociologist of Knowledge Say About 2+2=4? In: Ernest, P. (Hg.): Mathematics, Education and Philosophy: An International Perspective. London: Falmer, S. 21-32. 
Booysen, F. (2002): An Overview and Evaluation of Composite Indices of Development. In: Social Indicators Research 59, S. 115-151.

Burchell, S./Clubb, C./Hopwood, A.G./Hughes, J./Nahapiet, J. (1980): The Roles of Accounting in Organizations and Society. In: Accounting, Organizations and Society 5, S. 5-27.

Caiden, N./Wildavsky, A. (1974): Planning and Budgeting in Poor Countries. New York: Wiley.

Chua, W.F. (1986): Radical Developments in Accounting Thought. In: The Accounting Review 61, S. $601-632$.

Chua, W.F. (1995): Experts, Networks and Inscriptions in the Fabrication of Accounting Images: A Story of the Representation of Three Public Hospitals. In: Accounting, Organizations and Society 20, S. 111-145.

Cooper, D. J./Greenwood, R./Hinings, B./Brown, J. L. (1998): Globalization and Nationalism in a Multinational Accounting Firm: The Case of Opening New Markets in Eastern Europe. In: Accounting, Organizations and Society 23, S. 531-548.

Covaleski, M.A./Dirsmith, M.W. (1986): The Budgetary Process of Power and Politics. In: Accounting, Organizations and Society 11, S, 193-214.

Ferguson, J. (1990): The Anti-Politics Machine: 'Development', Depoliticization, and Bureaucratic Power in Lesotho. Cambridge: Cambridge University Press.

Ferguson, J. (1994): The Anti-Politics Machine. 'Development' and Bureaucratic Power in Lesotho. In: The Ecologist 24, S. 176-181.

Friedland, R./Alford, R. R. (1991): Bringing Society Back in: Symbols, Practices and Institutional Contradictions. In: Powell, W.W./DiMaggio, P.J. (Hg.): New Institutionalism in Organizational Analysis. Chicago; London: University of Chicago Press, S. 232-266.

Grusky, S. (2003): Water Privatization Myths: Broken Promises and Social Turmoil. Oxford: Prinwass.

Gustavsson, E. (2000): From a Hotel Guest to the '3.4. satisfied' Customer. In: Kalthoff, H./Rottenburg, R./Wagener, H.-J. (Hg.): Facts and Figures. Economic Representations and Practices. Marburg: Metropolis, S. 175-204.

Hacking, I. (1990): The Taming of Chance. Cambridge: Cambridge University Press.

Harper, R.H.R. (1998): Inside the IMF: An Ethnography of Documents, Technology and Organisational Action. San Diego/London: Academic Press.

Hopwood, A.G. (1987): The Archaeology of Accounting Systems. In: Accounting, Organizations and Society 12 , S. 207-234.

Hopwood, A.G./Miller, P. (Hg.) (1994): Accounting as Social and Institutional Practice. Cambridge: Cambridge University Press.

Hunt, H.G./Hogler, R.L. (1990): Agency Theory as Ideology: A Comparative Analysis Based on Critical Legal Theory and Radical Accounting. In: Accounting, Organizations and Society 15, S. 437-454.

Jackall, R. (1988): Moral Mazes. The World of Corporate Managers. New York/Oxford: Oxford University Press.

Jang, Y. S. (2006): Transparent Accounting as a World Societal Rule. In: Drori, G.S./Meyer, J. W./Hwang, H. (Hg.): Globalization and Organization: World Society and Organizational Change. Oxford: Oxford University Press, S. 167-195.

Kalthoff, H./Rottenburg, R./Wagener, H.-J. (Hg.) (2000): Facts and Figures. Economic Representations and Practices. Marburg: Metropolis.

Kieserling, A. (2003): Makropolitik, Mikropolitik, Politik der Protestbewegung. In: Nassehi, A./Schroer, M. (Hg.): Der Begriff des Politischen. Baden-Baden Nomos, Soziale Welt Sonderband, S. 419-439.

Latour, B. (1987): Science in Action: How to Follow Scientists and Engineers Through Society. Cambridge, Mass.: Harvard University Press.

Latour, B. (1990): Drawing Things Together. In: Lynch, M./Woolgar, S. (Hg.): Representation in Scientific Practice, Cambridge, Mass.: MIT Press, S. 19-68.

Luhmann, N. (1967): Soziologische Aufklärung. In: Soziale Welt 18, S. 97-123.

Luhmann, N. (1972): Knappheit, Geld und die bürgerliche Gesellschaft. In: Jahrbuch für Sozialwissenschaften 23 , S. 187-210. 
Luhmann, N. (1973): Zweckbegriff und Systemrationalität. Über die Funktion von Zwecken in sozialen Systemen. Frankfurt/M.: Suhrkamp.

Luhmann, N. (1981): Organisation im Wirtschaftssystem. In: Ders.: Soziologische Aufklärung 3. Opladen: Westdeutscher Verlag, S. 390-414.

Luhmann, N. (1988): Geld als Kommunikationsmedium: Über symbolische und diabolische Generalisierungen. In: Ders.: Die Wirtschaft der Gesellschaft. Frankfurt/M.: Suhrkamp, S. 230-271.

Luhmann, N. (1997): Die Gesellschaft der Gesellschaft. Frankfurt/M.: Suhrkamp.

Macintosh, N.B./Shearer, T./Thornton, D.B./Welker, M. (2000): Accounting as Simulacrum and Hyperreality: Perspectives on Income and Capital. In: Accounting, Organizations and Society 25 , S. $13-50$.

Mauss, M. (1990): Die Gabe. Form und Funktion des Austauschs in archaischen Gesellschaften. Frankfurt/M:: Suhrkamp.

Megginson, W.L./Netter, J.M. (2001): From State to Market. A Survey of Empirical Studies on Privatization. In: Journal of Economic Literature 39, S. 321-389.

Meltsner, A. (1976); Policy Analysts in the Bureaucracy. Berkeley: University of California Press.

Merton, R. K. (1952): Manifest and Latent Functions. In: Schuler, E.A. et al. (Hg.): Readings in Sociology. New York: Thomas Y. Crowell, S. 392-402.

Meyer, J.W. (1986): Social Environments and Organizational Accounting. In: Accounting, Organizations and Society 11, S. 345-356.

Morgan, G. (1988): Accounting as Reality Construction: Towards a New Epistemology for Accounting Practice. In: Accounting, Organizations and Society 13, S. 477-485.

Munro, R. (1993): Just When You Thought It Safe to Enter the Water: Accountability, Language Games and Multiple Control Technologies. In: Accounting, Management and Information Technology 3, S. 249-271.

Musto, S. A. (1987): Die hilflose Hilfe: Ansätze zu einer Kritik der manipulativen Vernunft. In: Schwefel, D. (Hg.): Soziale Wirkungen von Projekten in der Dritten Welt, Baden-Baden: Nomos, S. 419-503.

Neu, D./Ocampo, E./Ponce de León, O. G./Zepeda, M. F. (2002): Facilitating Globalization Processes: Financial Technologies and the World Bank. In: Accounting Forum 26, S. 271-290.

Neu, D./Ocampo, E. (2006): Doing Missionary Work: The World Bank and the Diffusion of Financial Practices. In: Accounting, Organizations and Society, in press.

Neuberger, O, (2001): Im Labyrinth der Daten und Interessen - Controlling als organisierte Vertragsüberwachung. In: Fischer, H. (Hg.): Unternehmensführung im Spannungsfeld zwischen Finanzund Kulturtechnik. Hamburg: Kovac, S. 173-194.

Pentland, B.T. (1993): Getting Comfortable with the Numbers: Auditing and the Micro-Production of Macro-Order. In: Accounting, Organizations and Society 18, S. 605-620.

Pinch, T./Ashmore, M./Mulkay, M. (2000): Cost-benefit Analysis in Practice: Option Appraisal in the UK National Health Service and the Politics of Rational Decision-making. In: Kalthoff, H./Rottenburg, R./Wagener, H.-J. (Hg.): Facts and Figures. Economic Representations and Practices. Marburg: Metropolis, S. 87-116.

Porter, T.M. (1995): Trust in Numbers: The Pursuit of Objectivity in Science and Public Life. Princeton, N.J.: Princeton University Press

Power, M. (1992): The Politics of Brand Accounting in the United Kingdom. In: European Accounting Review 1, S. 39-68.

Power, M. (1993): The Politics of Financial Accounting. In: The Political Quarterly 64, S. 272-284.

Radcliffe, V.S. (1999): Knowing Efficiency: The Enactment of Efficiency in Efficiency Auditing. In: Accounting, Organizations and Society 24, S. 333-362

Robson, K. (1991): On the Arenas of Accounting Change: The Process of Translation. In: Accounting, Organizations and Society 16, S. 547-570.

Robson, K. (1992): Accounting Numbers as ,Inscriptions": Action at a Distance and the Development of Accounting. In: Accounting, Organizations and Society 17, S. 685-708. 
Robson, K. (1993): Accounting Policy Making and 'Interest': Accounting for Research and Development. In: Critical Perspectives on Accounting 3, S. 1-27.

Rose, N. (1990): Governing the Soul. London: Routledge.

Rose, N. (1991): Governing By Numbers: Figuring Out Democracy. In: Accounting, Organizations and Society 16, S. 673-692.

Rottenburg, R. (2001): Kultur der Entwicklungszusammenarbeit mit Afrika. In: Wippel, S./Cornelssen, I. (Hg.): Entwicklungspolitische Perspektiven im Kontext wachsender Komplexităt. Festschrift für Prof. Dr. Dieter Weiß, Bonn: Weltforum, S. 349-377.

Rottenburg, R. (2002): Weit hergeholte Fakten. Eine Parabel der Entwicklungshilfe. Stuttgart: Lucius und Lucius.

Scheytt, T. (2005): Management Accounting from a Systems-Theoretical Perspective. In: Becker, K.H./Seidl, D. (Hg.): Niklas Luhmann and Organization Studies. Copenhagen: Copenhagen Business School Press, S. 386-404.

Stadler, L./Hoering, U. (2003): Das Wasser-Monopoly. Von einem Allgemeingut und seiner Priyatisierung. Zürich: Rotpunktverlag.

Tinker, T./Lehman, C./Neimark, M. (1988): Bookkeeping for Capitalism: The Mystery of Accounting for Unequal Exchange, In: Mosco, V./Wasko, J. (Hg.): The Political Economy of Information. Madison: University of Wisconsin Press, S. 188-216.

Ugaz, C. (2002): Liberalisation of Utilities Markets and Children's Right to Basic Services: Some Evidence from Latin America. Oxford: Prinwass.

Vollmer, H. (2004): Funktionen und Folgen organisierten Rechnens. In: Zeitschrift für Soziologie 33, S. $450-470$.

Vollmer, H. (2007): How to Do More With Numbers. Elementary Stakes, Framing, Keying, and the Three-dimensional Chracter of Numerical Signs. In: Accounting, Organizations and Society (im Erscheinen).

Vormbusch, U. (2004); Accounting. Die Macht der Zahlen im gegenwärtigen Kapitalismus. In: Berliner Journal für Soziologie 14, S. 33-50.

Wildavsky, A. (1964): The Politics of the Budgetary Process. Boston: Litte, Brown.

Wildavsky, A. (1975): Budgeting: A Comparative Theory of Budgetary Process. Boston: Little, Brown.

Wisner, B. (1989): Power and Need in Africa. Trenton: Africa World Press. 